\title{
Non-invasive assessment of pulmonary valve stenosis with a multigate pulsed Doppler system
}

\author{
S DE KNECHT, O DANIËLS, R S RENEMAN \\ From the Department of Paediatric Cardiology (Paediatric Clinic), University of Nijmegen, The Netherlands; and the \\ Department of Physiology, University of Limburg, Maastricht, The Netherlands
}

SUMMARY The width of the jet flow in the pulmonary artery was measured using a multigate pulsed Doppler system in a baby with severe pulmonary valve stenosis. The degree of stenosis estimated before operation agreed with the result obtained during surgery.

In paediatric cardiology there is a growing interest in non-invasive methods to detect cardiac disorders. The introduction of echocardiography, especially echo Doppler techniques, has added a new dimension by determining flow velocity in the heart cavities and blood vessels.

We have estimated the degree of pulmonary valve stenosis in a baby from the width of the jet stream in the pulmonary artery recorded using a multigate pulsed Doppler system. The result obtained before operation was compared with that estimated during surgery.

\section{Case report}

A baby boy was first seen in the paediatric department of a general hospital at the age of 1 week with a grade 3 (out of 6) pansystolic murmur in the third left intercostal space. At that time no specific clinical signs were present except for a slight tachypnoea noticed by the parents. Some weeks later he was referred to our clinic and echocardiography ( $M$ mode and sector scanner with echo Doppler; ATL Mark V) showed severe pulmonary valve stenosis without a ventricular septal defect. One month later, at the age of 2 months, the diagnosis was confirmed by cardiac catheterisation. The following results were obtained: pressure in the right ventricle was $80 / 0 \mathrm{~mm} \mathrm{Hg}$ and in the left ventricle $50 / 7 \mathrm{~mm} \mathrm{Hg}$. The foramen ovale was patent with a right to left shunt at this level. Angiography excluded a ventricular septal defect, and the right ventricular angiogram showed dome shaped pulmonary valves with a jet (Fig. 1).

Before operation, at the age of $2 \frac{1}{2}$ months, we estimated the pulmonary artery orifice by measuring the width of the jet flow in the pulmonary artery with a multigate pulsed Doppler system. We used a system with a $5 \mathrm{MHz}$ probe developed by Hoeks and others. ${ }^{1-3}$ The echo window was in the high left parasternal region, in the first or second intercostal space. The best location was detected by sector scanning (Fig. 2). The whole measuring distance of the 64 gates is $38.4 \mathrm{~mm}$ ( 1 gate $=0.6 \mathrm{~mm}$ ), and the width of the jet flow can be deduced from the number of sample segments with an abnormally high velocity pattern. The width of the jet stream was between 3.8 and $4.1 \mathrm{~mm}$, although this is an overestimate because the sound beam crosses the artery obliquely to obtain a Doppler signal.

Two days later a valvotomy was performed. Before valvotomy, however, a surgical probe was passed through the ostium: a probe of $3 \mathrm{~mm}$ in diameter could pass the ostium, while a $4 \mathrm{~mm}$ diameter probe could not.

\section{Discussion}

These findings indicate that the degree of pulmonary valve stenosis can be estimated from the width of the jet flow in the pulmonary artery. This is in agreement with the findings of Kececioglu-Draelos et al. ${ }^{4}$ that in severe stenosis the diameter of the jet flow distal to the stenosis is similar to that of the obstructive orifice. The possibility of measuring the jet flow diameter has already been investigated by Johnson et al. in 1973,5 but they used a single gate pulsed Doppler system in which the region sampled could be altered with a range knob. With a multigate pulsed Doppler system it is easier to explore the pulmonary artery and to find 


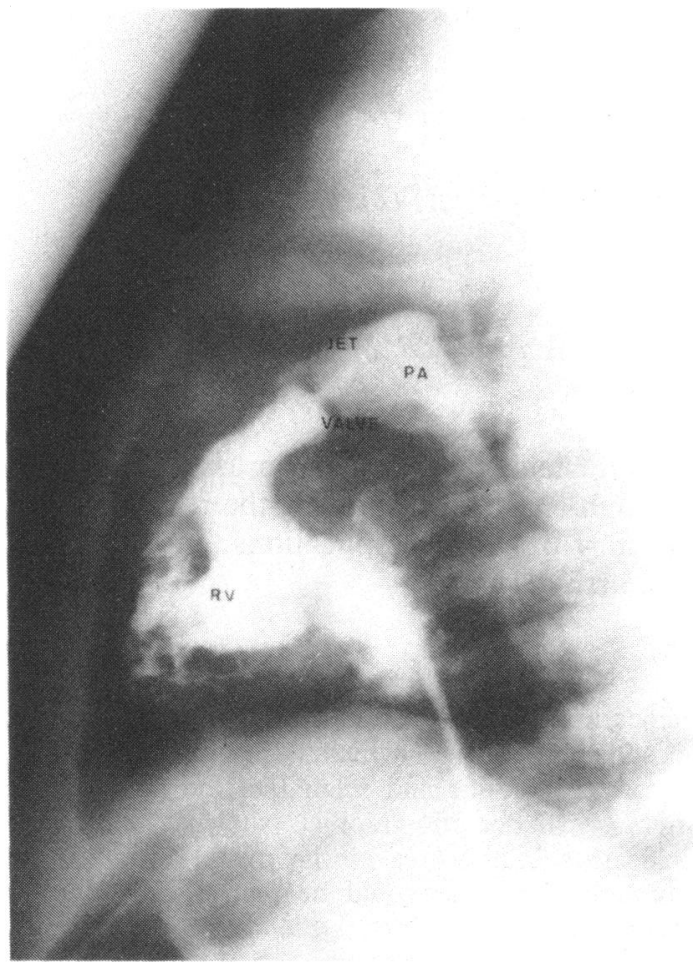

Fig. 1 Right ventricular angiocardiogram in a lateral view. The catheter is in the right ventricle $(R V)$, while the jet produced by the stenotic pulmonary valve is seen in the pulmonary artery $(P A)$.

the jet flow. Recently we connected the multigate pulsed Doppler system to the ATL Mark V sector scanner so that the site of velocity sampling could be localised accurately.

We thank Dr Ir A P G Hoeks and Dr J C W Hopman for their technical advice and assistance.

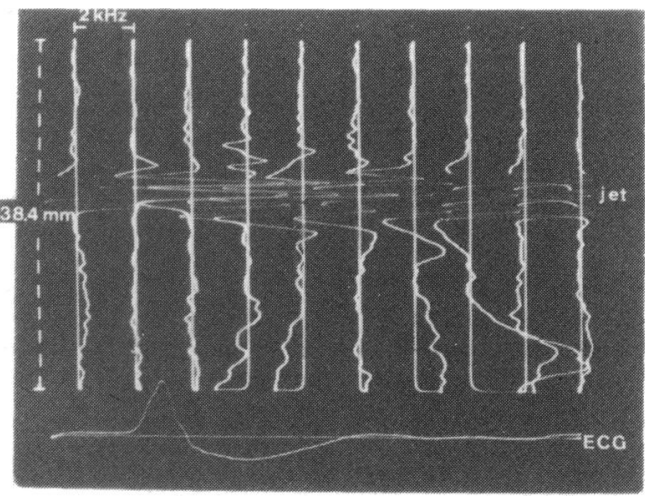

Fig. 2 Velocity profiles at discrete intervals during one cardiac cycle. The whole depth is $38.4 \mathrm{~mm}=64$ gates. The width of the jet can be calculated from the number of sample segments (gates) with an abnormally high velocity pattern.

\section{References}

1 Hoeks APG, Reneman RS, Peronneau PA. A multigate pulsed Doppler system with serial data processing. IEEE Trans Sonics Ultrasonics 1981; SU-28: 242-7.

2 Hoeks APG. On the development of a multigate Doppler system with serial data processing. Maastricht; The Netherlands: University of Limburg, 1982. Thesis.

3 Hoeks APG. Doppler measurement in heart chambers and ascending aorta; comparing single and multi-channel systems. In: Spencer MP, ed. Cardiac Doppler diagnosis. The Hague: M Nijhoff, 1983: 43-50.

4 Kececioglu-Draelos Z, Goldberg SJ, Areias J, Sahn DJ. Verification and clinical demonstration of the echo Doppler series effect and vortex shed distance. Circulation 1981; 63: 1422-9.

5 Johnson SL, Baker DW, Lute RA, Murray JA. Jet diameter in aortic stenosis, measured by Doppler echocardiography [Abstract]. Circulation 1973; 47 and 48 (suppl IV): 126.

Requests for reprints to Dr S de Knecht, Instituut voor Kindergeneeskunde, Geert Grooteplein Zuid 20, 6500 HB Nijmegen, The Netherlands. 